\title{
Inhibition of agonist-stimulated aldosterone production from adrenal zona glomerulosa cells by ANP is mediated by GC-A Stepan Gambaryan*1, Michaela Kuhn ${ }^{2}$ and Ulrich Walter ${ }^{1}$
}

\author{
Address: ${ }^{1}$ Institute of Clinical Biochemistry and Pathobiochemistry, University of Wuerzburg, 97078 Wuerzburg, Germany and ${ }^{2}$ Institute of \\ Physiology University of Wuerzburg, 97070 Wuerzburg, Germany \\ Email: Stepan Gambaryan* - gambaryan@klin-biochem.uni-wuerzburg.de \\ * Corresponding author
}

from 2nd International Conference of cGMP Generators, Effectors and Therapeutic Implications

Potsdam, Germany, 10-12 June, 2005

Published: 16 June 2005

BMC Pharmacology 2005, 5(Suppl I):PI9 doi:I0.II86/I47I-2210-5-SI-PI9

Atrial natriuretic peptide (ANP) is a potent inhibitor of agonist-induced aldosterone synthesis in zona glomerulosa (ZG) cells of the adrenal gland. Cellular ANP effects are mediated by two receptors: GC-A, a membrane guanylyl cyclase, and GC-C that lacks guanylyl cyclase activity. To identify which one of these two receptors is involved in inhibitory pathway of aldosterone production we developed a new model of isolation and primary culture of mouse ZG cells. Aldosterone concentration in the blood was not different in wild type and GC-A knockout mice. ANP (10 nM) significantly inhibited basal, forskolin, and Angiotensin II-stimulated aldosterone production from wild type mice, while in GC-A knockout mice ANP had no inhibitory effect on aldosterone production. Inhibition of forskolin or ACTH stimulated aldosterone production by ANP was connected with increased concentration of cGMP, stimulation of cGMP-stimulated phosphodiesterase (PDE2), and decrease of intracellular cAMP content. IBMX and the PDE2-selective inhibitor MEP-1 completely prevented ANP inhibitory effect on cAMP-stimulated aldosterone production. In contrast, ANP-dependent inhibition of Ang II-stimulated aldosterone production was not connected with PDE2 or PKG activity indicating that other cGMP-dependent mediator(s)/mechanism(s) are involved in regulation of Ang II-stimulated aldosterone production from ZG cells by cGMP.

\section{Acknowledgements}

This work was supported by the Deutsche Forschungsgemeinschaft grant WA 366 